\title{
YIELD MANAGEMENT EM INSTITUIÇÕES DE ENSINO SUPERIOR: UM ESTUDO DE CASO NA MODALIDADE DE ENSINO A DISTÂNCIA DE UMA UNIVERSIDADE COMUNITÁRIA
}

\section{RESUMO}

O aumento da concorrência entre as instituições de ensino superior tem impulsionado-as a buscar maneiras de aumentar a demanda para melhorar seus resultados. O Yield Management vem ao encontro dessa necessidade como uma ferramenta de apoio a gestão que trabalha o equilíbrio entre a demanda e a oferta com o objetivo de maximizar receita. Dentro deste contexto, o presente estudo visa analisar como o Yield Management pode ser utilizado para otimizar a receita de um curso de graduação a distância de uma instituição de ensino superior comunitária. Para tanto, o estudo envolve a aplicação de pesquisa exploratória, de abordagem qualitativa na forma de estudo de caso. A coleta de dados foi realizada por meio de entrevistas. A partir dos resultados obtidos constatou-se: i) que a instituição possui os requisitos necessários para a aplicação da ferramenta Yield Management; ii) que existe uma diminuição no número de matrículas nos últimos dois semestres e que as estratégias utilizadas pelo curso não estão sendo suficientes para o preenchimento das vagas ofertadas; iii) que o modelo proposto permitirá que o curso tenha estratégias voltadas para o equilíbrio entre a oferta e demanda, aumentando o número de matrículas e consequentemente tornando-se mais competitivo.

Palavras-chave: Gerenciamento de Receitas; Precificação; Instituições de Ensino Superior; Avaliação do Desempenho.

\section{YIELD MANAGEMENT IN HIGHER EDUCATION INSTITUTIONS: A CASE STUDY IN THE DISTANCE OF EDUCATION FOR COMMUNITY UNIVERSITY}

\begin{abstract}
Increased competition between higher education institutions has driven them to seek ways to increase demand to improve your results. Yield Management meets this need as a support management tool that works the balance between demand and supply in order to maximize revenue. Within this context, this study aims to analyze how the Yield Management can be used to optimize the revenue of an undergraduate degree at a distance of a top community college. Therefore, the study involves the application of exploratory research, qualitative approach in the form of case study. Data collection was conducted through interviews. From the results found that: i) the institution has the necessary requirements for the application of Yield Management Tool; ii) there is a decrease in enrollment in the past two semesters and that the strategies used by the course are not enough to fill the vacancies offered; iii) that the proposed model will allow the course has strategies for the balance between supply and demand, increasing enrollment and thus becoming more competitive.
\end{abstract}

Keywords: Revenue Management; Pricing; Higher Education Institutions; Performance Evaluation. 


\section{YIELD MANAGEMENT EN INSTITUCIONES DE EDUCACIÓN SUPERIOR: UN ESTUDIO DE CASO EN LA DISTANCIA DE EDUCACIÓN PARA LA COMUNIDAD UNIVERSITARIA}

\section{RESUMEN}

El aumento de la competencia entre las instituciones de educación superior les ha impulsado a buscar formas de aumentar la demanda para mejorar sus resultados. Yield Management responde a esta necesidad como una herramienta de gestión de apoyo que trabaja el equilibrio entre la oferta y la demanda con el fin de maximizar los ingresos. Dentro de este contexto, el presente estudio pretende analizar cómo el Yield Management se puede utilizar para optimizar los ingresos de un título de grado a una distancia de un colegio universitario superior. Por lo tanto, el estudio consiste en la aplicación de la investigación exploratoria, enfoque cualitativo en la forma de estudio de caso. La recolección de datos se realizó a través de entrevistas. A partir de los resultados encontró que: i) la entidad tiene los requisitos necesarios para la aplicación de la herramienta de gestión de rendimiento; ii) hay una disminución de la matrícula en los últimos dos semestres y que las estrategias utilizadas por el curso no son suficientes para cubrir las vacantes que se ofrecen; iii) que el modelo propuesto permitirá que el curso tiene estrategias para el equilibrio entre la oferta y la demanda, el aumento de la matrícula y convirtiéndose así en más competitivo.

Palabras clave: Gestión de Ingresos; La Fijación de Precios; Las Instituciones de Educación Superior; Evaluación del Desempeño.

Patrícia Schmidt Hahn de Lima ${ }^{1}$ Carlos Rogerio Montenegro de Lima $^{2}$ Ibsem Agrello Dias ${ }^{3}$ Marcus Vinicius Andrade de Lima ${ }^{4}$

\footnotetext{
${ }^{1}$ Mestre em Administração pela Universidade do Sul de Santa Catarina - UNISUL. Diretora Acadêmica na Faculdade Católica de Santa Catarina. Brasil. E-mail: patyhlima@gmail.com

${ }^{2}$ Doutor em Engenharia de Produção pela Universidade Federal de Santa Catarina - UFSC. Professor do Programa de Pós-Graduação em Administração - Mestrado em Administração - da Universidade do Sul de Santa Catarina - UNISUL. Brasil. E-mail: calmontenegro@gmail.com

3 Mestre em Administração pela Universidade do Sul de Santa Catarina - UNISUL. Brasil. E-mail: ibsem.dias@gmail.com

${ }^{4}$ Doutor em Engenharia de Produção pela Universidade Federal de Santa Catarina - UFSC. Professor da Universidade Federal de Santa Catarina - UFSC. Brasil. E-mail: marcus.lima@ cse.ufsc.br
} 


\section{INTRODUÇÃO}

$\mathrm{O}$ século $\mathrm{XX}$ foi palco de grandes desafios para as organizações. À medida que o mundo dos negócios foi se interligando, as exigências ficaram mais dinâmicas levando as pessoas e organizações a refletirem sobre as competências necessárias a sobrevivência num mercado competitivo. Este cenário não foi diferente para o segmento educacional no Brasil, devido a fatores como a expansão e a consolidação do setor privado até a década de 80 e a medidas governamentais e procedimentos instituídos a partir da década de 90 para funcionamento das escolas de nível superior (NASSIF; HANASHIRO, 2003).

Segundo os parâmetros divulgados pelo Instituto Nacional de Estudos e Pesquisas Educacionais Anísio Teixeira - Inep o Brasil passou por uma mudança na Educação Superior a partir dos anos 90, quando a Lei de Diretrizes e Bases da Educação flexibilizou a entrada de iniciativas privadas neste setor. Os dados mostram que o país passou de 894 Instituições de Ensino Superior (IES) em 1995 para 2.416 em 2012. Ressalta-se que das 2.416 IES, 2.112 são instituições privadas (BRASIL, INEP, 2014).

Diante dos dados apresentados, observa-se que o país possui um mercado de educação evidenciado pelo crescimento e pela concorrência acirrada das Instituições de Ensino Superior (IES), principalmente quando o foco é a rede privada de ensino, forçando estas instituições a desenvolverem novas habilidades e em consequência novas formas de abordagem de alunos, a fim de se tornarem mais eficientes no processo de atração dos mesmos (ALESSIO et al., 2010).

Desta forma, muitos são os desafios enfrentados pelos gestores que a cada dia se reinventam com o intuito de criar novas estratégias para atrair estudantes para as salas de aula. Dentre os desafios encontrados pelo gestor está a gestão da capacidade em um sistema prestador de serviços, pois a forma como a capacidade é gerida pode implicar na rentabilidade, ou não, de um negócio. Isto porque o dimensionamento da capacidade afeta o desempenho da empresa, pois tem impacto nos investimentos e nos custos operacionais. Em função de certas características dos serviços, como a intangibilidade e a simultaneidade entre produção e consumo, a capacidade dos sistemas de serviços é perecível, não pode ser estocada (FIGUEIREDO; ESCOBAR, 2004). No caso de uma IES, as vagas que não são preenchidas não podem ser estocadas para o próximo semestre, fato esse que acaba acarretando perdas significativas para a instituição.

Outro fator importante é compreender como funciona a demanda futura. Uma das ferramentas que procura trabalhar a demanda, principalmente em empresas de serviço, e que tem obtido um crescente destaque, especialmente internacional, é o Gerenciamento de Receitas (Revenue Management ou Yield Management). O principal objetivo do Yield
Management (YM), de acordo com uma definição tradicional, é extrair o máximo de receita possível de determinada capacidade existente, que nas empresas onde é comumente utilizado, geralmente é fixa (GU; CANEEN, 1998).

O YM originou-se na indústria aérea americana após a sua desregulamentação em 1978. Esta desregulamentação foi um conceito revolucionário que permitiu a liberdade na indústria da aviação no desenvolvimento de estratégias de marketing e de preços. Estas condições de mercado forçaram a gestão de companhias aéreas a se concentrar em novas abordagens para a gestão do produto perecível das companhias aéreas - o assento dos passageiros em cada voo (WANG; BOWIE, 2009).

Wang e Bowie (2009) mostram que o Yield Management (YM) tem sido amplamente adotado por uma série de setores com limitações de capacidade na indústria de serviços nas últimas duas décadas. Exemplos na literatura sobre hospitalidade incluem Orkin (1988), Kimes (1989), Brotherton e Mooney (1992), Weatherford e Kimes (2001); na área da saúde, Kimes (1989); em centros de convenções, Hartley e Rand (1997); em parques temáticos, Goulding e Leask (1997); em cruzeiros marítimos, Hoseason e Johns (1998); e no golfe, Kimes (2000). No entanto, considerando-se a extensa pesquisa em gestão de receitas nestes setores, há um número muito limitado de pesquisas do YM no setor da educação.

Nesse panorama, visando à obtenção de informações que possam ser aproveitadas pelas IES de maneira estratégica para a captação e retenção de alunos, torna-se importante investigar a relevância do YM nesse processo, avaliando a possibilidade de sua implantação, assim como os possíveis resultados de otimização dos lucros gerados pela previsão do comportamento da demanda. Nesse sentido, este estudo busca responder a seguinte pergunta de pesquisa: Como o Yield Management pode ser utilizado para otimizar a receita da modalidade de ensino a distância de uma IES comunitária?

Para responder a esta pergunta, o objetivo geral deste estudo consiste em propor a utilização do Yield Management como ferramenta para a otimização de receitas da modalidade de ensino a distância de uma IES comunitária. Para consecução do objetivo geral foram estabelecidos os seguintes objetivos específicos: (i) Contextualizar o problema da instituição de ensino, foco do estudo, entendendo o ambiente e evidenciando as formas de comercialização dos seus serviços; (ii) Analisar se a modalidade de ensino a distância da instituição atende os requisitos necessários para a adoção do Yield Management; e (iii) Propor os passos para a implantação do YM na instituição.

Este trabalho está estruturado em cinco capítulos: introdução, referencial teórico, aspectos metodológicos da pesquisa, resultados e análises de dados e considerações finais. 


\section{REFERENCIAL TEÓRICO}

A competitividade das IES privadas está associada, entre outros fatores, a suas estratégias de gerenciamento de receitas e sua disseminação e operacionalização pelos gestores da organização. É, portanto, fundamental para o sucesso da IES que estas sejam entendidas e mensuradas em forma inteligível e não ambígua para permitir o seu contínuo monitoramento e aperfeiçoamento.

Os eixos que explicam o conteúdo apresentado nesta definição no entendimento do pesquisador são: Avaliação de Desempenho, Gerenciamento de Receitas, Ensino Superior - Recrutamento, Ingressos e Mensalidades.

\subsection{Avaliação de desempenho}

A avaliação de desempenho tem sido utilizada a milênios como instrumento gerencial. Até a década de 1960, os meios de produção se baseavam estritamente em ativos e força de trabalho. O gerenciamento e controle centravam na otimização do uso destes ativos em conjunção com o trabalho braçal e as organizações competiam apenas por preço e qualidade (HAYS E PISANO, 1994). A partir de então, as dimensões, agilidade, flexibilidade e inovação, em conjunto com qualidade e preço, passam a estabelecer o grau de sucesso das organizações. Surgem novas formas de gestão alicerçadas em instrumentos de avaliação de desempenho que centram seus esforços em como utilizar os meios existentes para alcançar os objetivos estratégicos.

Associado a esta visão emergiram inúmeras propostas para mensurar o desempenho das organizações, dentre elas: i- Performance Measurement Process - Nevada Family Quality Fórum; iiDeveloping Performance Indicators (MIDWINTER, A.,1994), A systemic Approach - Sandia National Laboratories (SZTIPANOVITS, J.; KARSAI, G.,1997); iii- Developing Performance Metrics University of California Approach; Balanced Scorecard de Kaplan e Norton (KAPLAN; NORTON, 1992, 1996, 2004); iv- Baldrige (GARVIN, D. A.,1991); vStrategic Measurement, Analysis, and Reporting Technique SMART - Performance Pyramid (CROSS; LYNCH, 1988), Vi- Sinais Vitais (HRONEC, 1994), vii- Performance Prism (NEELY; ADAMS; KENNERLEY, 2002), viii - Metodologia Multicritério em Apoio à Decisão - MCDA (ROY, 1985; ROY, 1993; LANDRY, 1995; KEENEY, 1992; BANA E COSTA, 1993).

A gestão organizacional neste contexto é definida como o conjunto de atividades utilizadas para observar, planejar, organizar, operacionalizar, monitorar e aperfeiçoar a melhoria contínua de processos e produtos. E, uma das formas de realizar a gestão é por meio da construção de conhecimento no gestor sobre esse contexto. Esse processo de geração de conhecimento é neste trabalho tratado como avaliação de desempenho e tem a seguinte definição:

O processo para construir conhecimento no decisor, a respeito do contexto específico que se propõe avaliar, a partir da percepção do próprio decisor por meio de atividades que identificam, organizam, mensuram ordinalmente e cardinalmente, integram e permitem visualizar o impacto das ações e seu gerenciamento. (ENSSLIN et al., 2010).

\subsection{Ensino superior - recrutamento, ingressos e mensalidades}

Montgomery (2002) constata que a elasticidade da escolha de uma escola de negócios de pós-graduação no que diz respeito à mensalidade é baixa e a localização é um poderoso determinante de escolha da escola. Por outro lado, diversos outros estudos apresentados a seguir demonstram relação entre preço e demanda.

$\mathrm{Fu}$ (2010) desenvolveu e estimou de forma estruturada um modelo de equilíbrio para o mercado de ensino superior que incorpora mensalidades, inscrições, admissões e matrículas. $\mathrm{O}$ autor concluiu que as faculdades tendem a cobrar menos do que poderiam quando as políticas de mensalidades não desempenham um papel estratégico na instituição. Além disso, a subprecificação é mais significativa para as faculdades com avaliação mais baixas nos rankings do que para melhores avaliadas. Epple, Romano e Sieg (2006) apresentam um modelo de equilíbrio do mercado para o ensino superior que prevê simultaneamente a seleção de alunos em instituições de ensino superior, auxílio financeiro, despesas e resultados educacionais. Mostram que o modelo dá origem a uma estrita hierarquia de faculdades que diferem pela qualidade do ensino prestado aos alunos.

Antons e Maltz (2006) mostram que algumas universidades oferecem ajuda financeira a uma grande parte dos seus novos alunos, tanto como um meio de satisfazer a necessidade financeira dos estudantes como uma ferramenta de recrutamento. A utilização de auxílios financeiros fornece uma poderosa alavanca para as admissões, mas estas decisões também possuem implicações financeiras. Friedel e Thomas (2013) apresentam como um diferencial nas mensalidades é usado por faculdades comunitárias norte-americanas com a finalidade de criar maior acesso para os alunos.

Buss, Parker e Rivenburg (2004) em pesquisa realizada em um grupo de faculdades de artes liberais concluíram que os estudantes que não necessitam de auxílio financeiro têm uma elasticidade próxima da unidade, o que implica que o aumento de $1 \%$ na mensalidade implica na diminuição de $1 \%$ no resultado das matrículas. Analisando os beneficiários de auxílio financeiro, a pesquisa sugere que os valores de mensalidades, alojamento e alimentação afetam 
negativamente o resultado de inscrição desse grupo de estudantes, enquanto auxílio financeiro e empréstimos aumentam a probabilidade de matrícula. Usando dados do ranking das escolas de direito norte-americanas no período 1996-2003, Sauder e Lancaster (2006) concluíram que a classificação das escolas tem efeito significativo sobre as decisões de futuros alunos e as decisões que as escolas fazem em seus processos de admissão. Por sua vez, para Nurnberg, Schapiro e Zimmerman (2012), a qualidade, o preço líquido para um aluno em particular enfrenta (o preço menos o auxílio financeiro institucional), a etnia do candidato e origem geográfica, além dos seus interesses artísticos, esportivos e acadêmicos, são fatores que indicam fortes indícios se o aluno se matriculará ou não em uma IES.

Hillman (2012) mostra que durante a última década, o auxílio financeiro institucional (ou "descontos nas mensalidades") foi o item que mais cresceu nos orçamentos operacionais dentro da maioria das faculdades e universidades públicas norteamericanas devido à uma mudança na estrutura dos fluxos de receitas, com mensalidades e taxas substituindo as dotações estaduais como fonte de financiamento. Demonstra que este auxílio financeiro tem gerado receita adicional para estas IES e conclui que essa relação só é sustentável até um certo ponto.

\subsection{Gerenciamento de receitas}

A gestão de receitas (revenue management ou yield management) é uma forma sofisticada de gestão da oferta e da procura que equilibra as estratégias de preços com gestão de inventários. É essencialmente o processo de alocação do tipo certo de capacidade para o cliente certo, na hora certa, pelo preço certo. Centrase a organização em maximizar a lucratividade por meio da aplicação de táticas disciplinados para prever o comportamento do consumidor e controlar a disponibilidade de estoque em cada nível de preços (WIRTZ, 2003). Este assunto tem sido tratado na literatura de diversas formas.

Do ponto de vista dos pesquisadores Bobb e Veral (2008), estudos de gerenciamento de receitas se concentraram em pesquisas sobre overbooking com o primeiro modelo estatístico introduzido por Beckmann e Bobkowski em 1958. Posteriormente, o trabalho pioneiro de Littlewood em 1972 apresentou modelos de maximização de receitas por meio de sistemas de limites de reserva e controle de inventários. Historicamente, a gestão das receitas começou como uma função de operações, focando apenas na atribuição de capacidade a partir das estimativas de demanda. $\mathrm{Na}$ década de 1960, a American Airlines começou a usar modelos de Pesquisa Operacional para as decisões de gestão de receitas. Na década de 1980, a gestão das receitas se tornou um sistema robusto e eficaz trazendo uma solução prática para resolver os problemas de capacidade e demanda. $\mathrm{Na}$ perspectiva dos profissionais da área, na década de 1990 ocorreu não só a proliferação de aplicações de gestão de receitas na indústria aérea, mas também o crescimento nas indústrias hoteleira, de hospitalidade e viagens, que enfrentam problemas semelhantes.

Jallat e Ancarani (2008) mostram como a gestão de receitas (YM) e preços dinâmicos, que se originou no setor aéreo, estão se difundindo em outros setores de serviços. Demonstram que estas técnicas podem ser proveitosamente aplicadas a setores de telecomunicações e similares. Garrow et al. (2006) apresentam as práticas de gerenciamento de receitas e precificação nos diversos ramos de atividade que tem utilizado a YM - companhias aéreas, hotéis, locadoras de veículos, cruzeiros, aluguéis de imóveis, fabricantes de aeronaves, varejo, distribuição, e-mail marketing, viagens, logística, esportes, fornecedores de software, entre outros.

Garrow e Ferguson (2009) afirmam que com a crise econômica norte-americana pós 11 de setembro de 2008, muitas empresas estão utilizando abordagens de gerenciamento de receitas (YM) para entender melhor os direcionadores de demanda e ofertas de descontos que oferecem valor para segmentos específicos de clientes.

Noone, McGuire e Rohlfs (2011) mostram que recentemente a YM começou a evoluir a partir de uma orientação tática para um papel mais estratégico dentro das organizações hoteleiras que engloba marketing, vendas e estratégia de canais. Com essa evolução surgiu um conjunto mais amplo de responsabilidades em outras áreas, incluindo precificação, gestão do fluxo de receitas, e uma abordagem centrada no cliente para o desenvolvimento de demanda.

$\mathrm{Ng}$ (2006) propõe duas estratégias para auxiliar as empresas a melhorar as receitas e reduzir a incerteza: a diferenciação de serviço dinâmico e a autoseleção. Pela diferenciação de serviço dinâmico, os atributos de serviços são alterados dinamicamente de acordo com as preferências dos consumidores. Propõe também que as empresas empreguem a segmentação baseada na auto-seleção, oferecendo aos consumidores uma gama de opções com preços diferentes, sem a necessidade de pré-determinar os segmentos. Gallego e Phillips (2004) sugerem que oferecer produtos flexíveis/substitutos tem potencial tanto para aumentar a demanda como para melhorar o equilíbrio entre demanda e capacidade.

Schwartz (2006) discute alguns fatores relacionados com as reservas controlados pelos hotéis (como o tamanho da multa por cancelamento de uma reserva), demonstrando como eles afetam as faixas de preços e a estratégias de reservas. A análise indica que a eficácia e sucesso das políticas de comercialização e de preços depende da distribuição dos consumidores no plano preço/utilidade. Collins e Parsa (2006), por sua vez, mostram que as estratégias de preço final podem ser utilizadas para sinalizar valor ou qualidade. Demonstram que a medida que a diária média diminui, a prática de preços muda de dólar, para dólar e centavos. 
Com recursos tecnológicos e utilizando técnicas matemáticas, Bobb e Veral (2008) afirmam que a ascendência no mercado tem historicamente estado com os prestadores de serviços. No entanto, aplicativos baseados na web estão mudando lentamente este poder para o consumidor. Inicialmente desenvolvido como uma ferramenta de marketing para a precificação de passagens aéreas, inúmeras aplicações de Revenue Management (RM) atuais podem se beneficiar de ferramentas contábeis que auxiliam a avaliar e monitorar se o RM aumentará o resultado operacional (HUEFNER; LARGAY, 2008). Bitran e Caldentey (2003) formulam um modelo para definir dinamicamente a lista e a quantidade de produtos e seus preços correspondentes para maximizar a receita total ao longo do horizonte de venda. Tokman, Davis e Lemon (2007) apresentam como a reaquisição de clientes fornece às empresas elevados benefícios financeiros e de melhoria de serviços.

Wirtz et al. (2003) alertam para possíveis conflitos entre a estratégia de orientação para o cliente e a gestão das receitas e sugerem uma série de ações para evitar prejuízos para o sucesso no longo prazo. Lowe e Alpert (2007) investigaram a percepção de preços de novos produtos e concluíram que os consumidores tendem a evocar o conceito de preço justo para novas categorias de produtos e do conceito de preço esperado para as categorias existentes. Para Xia, Monroe e Cox (2004), Mathies e Gudergan (2007), a percepção de injustiça no preço pode levar a consequências negativas para o vendedor. Wang e Bowie (2009) afirmam que os benefícios da gestão de receitas recompensam principalmente a empresa, enquanto a longo prazo o desenvolvimento de relacionamento B2B sofre as consequências de curto prazo do comportamento oportunista da empresa.

Choi e Mattila (2005) sugerem que, para garantir os benefícios a longo prazo de práticas de RM, deve-se avaliar cuidadosamente a percepção dos clientes da equidade destas práticas e tomar as medidas necessárias para minimizar o potencial impacto negativo nos negócios devido a uma possível percepção de injustiça. Assim, Choi e Mattila (2005), Wirtz e Kimes (2007) propõem que os clientes sejam informados sobre as práticas de preços diferentes a fim de melhorar a percepção de equidade.

Após três décadas de utilização cada vez maior da gestão de receitas (RM), o que se pode esperar para o futuro? Milla e Shoemaker (2008) discutem as tendências futuras na gestão de receitas (RM) na indústria hoteleira com base em entrevistas com líderes da indústria. A integração do comportamento e preferências dos clientes com estratégias de marketing e de preços parece ser uma grande tendência para o futuro. Cross, Higbie e Cross (2009) concluem que conceitos como gestão de receitas centrada no cliente e planejamento de demanda da empresa baseiam-se, em parte, na ideia de que o futuro da gestão de receitas é adicionar uma perspectiva de longo prazo para uma disciplina que tem até agora focado no curto prazo. Esta ideia reflete a ampliação do papel estratégico que a gestão das receitas está tendo, e que continuará a levar novas fronteiras para a disciplina.

\section{MÉTODO}

Esta seção apresenta os aspectos metodológicos da pesquisa de campo do presente estudo, incluindo o delineamento da pesquisa, os procedimentos metodológicos a serem empregados e o instrumento de intervenção Yield Management.

\subsection{Delineamento da pesquisa}

Esta pesquisa se estrutura a partir do Paradigma Interpretativista, pois compreende que a sociedade se estabelece de forma subjetiva, constituindo-se a partir da ação dos indivíduos que a compõem, construindo simbolicamente e socialmente sua própria realidade organizacional, ou seja, é um produto da experiência das relações entre sujeitos e/ou sujeito e objeto. O teórico social-interpretativista procura compreender o processo em que as múltiplas realidades compartilhadas surgem, se sustentam e se transformam (MORGAN, 2007, p.16).

A lógica de pesquisa é indutiva quando o pesquisador busca o conhecimento na observação da realidade empírica e o problema pesquisado ainda é inexplorado, trazendo novos esclarecimentos para o meio científico (RICHARDSON; PERES, 1985). Este trabalho tem lógica indutiva, pois o conhecimento gerado acontece a partir da visão particular do pesquisador a respeito da aplicação do instrumento Yield Management utilizado para a gestão de receitas em outras áreas e propostos para uma Instituição de Ensino Superior.

A diferença entre as abordagens está relacionada à perspectiva de análise, ou seja, "enquanto cientistas sociais que trabalham com estatística apreendem dos fenômenos apenas a região visível, ecológica, morfológica e concreta; a abordagem qualitativa aprofunda-se no mundo dos significados das ações e relações humanas, um lado não perceptível e captável em equações, médias e estatísticas" (DESLANDES, 1999, p. 22). Este trabalho tem abordagem qualitativa caracterizada à aplicação das entrevistas semiestruturadas, nas quais o entrevistado concede declarações que são utilizadas na análise e interpretação dos dados.

Quanto os objetivos, os estudos científicos são classificados em exploratórios, descritivos e causais (SAUNDERS; LEWIS; THORNHILL, 2003). A pesquisa será tratada, quanto ao objetivo, como uma pesquisa exploratória e descritiva. Exploratória, já que o fenômeno analisado tem sido ainda pouco investigado e há necessidade de ampliar a compreensão sobre como o Yield Management pode contribuir para otimizar receitas em instituições de ensino superior. 
A pesquisa descritiva, segundo Collins e Hussey (2005, p. 24), é a pesquisa que descreve o comportamento dos fenômenos. É usada para identificar e obter informações sobre as características de um determinado problema ou questão.

Para a realização da pesquisa apresentada, o presente estudo encontra-se evidenciado pela utilização de estudo de caso e do levantamento bibliográfico. Para Triviños (2011), o estudo de caso é categoria de pesquisa em que se analisa uma unidade com profundidade, ou seja, a característica da pesquisa dáse em razão da natureza e abrangência da unidade. Dessa forma, a pesquisa pode ser percebida como um estudo de caso diante da proposta de elaboração do estudo para um contexto único, específico - nesse caso, a realização de uma pesquisa envolvendo o Curso de Administração a Distância da Universidade Alfa (nome fictício).

No que se refere ao levantamento bibliográfico, Vergara (2010) pontua que a pesquisa bibliográfica é um estudo sistematizado, desenvolvido com base em material publicado em livros, revistas, meios eletrônicos e jornais. $\mathrm{O}$ método tem por objetivo fornecer instrumental analítico para a pesquisa. Triviños (2011) acrescenta que o levantamento bibliográfico é importante para uma pesquisa na medida em que os demais instrumentos, tais como o questionário e a entrevista, também utilizados para a coleta de informações, são iluminados pelos conceitos verificados na teoria. Desta forma, a pesquisa bibliográfica serviu de base para a revisão da literatura que teve por objetivo proporcionar a análise crítica de publicações selecionadas, no sentido de evidenciar as contribuições ofertadas pelo presente trabalho, bem como suas limitações.

O presente estudo envolverá a coleta de dados primários e secundários. A coleta de dados primários incluirá a aplicação de entrevista.

A coleta de dados terá início com a aplicação do método levantamento documental, que compreende a busca de dados secundários pela análise de materiais e documentos (impressos e eletrônicos) disponibilizados pela empresa, incluindo do website corporativo, publicações de mercado, documentos internos de marketing, material promocional/ publicitário e outros materiais/documentos internos (como comunicados internos, declaração de missão/ visão/ valores da empresa e documento com dados históricos da empresa e do curso estudado). A fonte será secundária quando possuir, ao menos, um nível de interpretação entre o fato e seu registro (COOPER; SCHINDLER, 2014). Dessa forma, podem ser consideradas secundárias as fontes obtidas por meio de levantamento bibliográfico.

A segunda etapa da coleta de dados envolverá a aplicação de entrevista, sendo que as mesmas serão aplicadas de forma individualizada (em um único momento) no ambiente da empresa. Os roteiros de entrevistas serão elaborados após a coleta de dados secundários considerando os objetivos específicos do estudo.

Conforme Yin (2001), a análise de dados consiste em examinar, classificar, categorizar e recombinar as evidências coletadas (sejam de natureza primária e secundária) para identificar conclusões, proposições e/ou hipóteses de pesquisa a serem testadas. Qualquer técnica de análise de dados, em última instância, significa uma metodologia de interpretação. Como tal, possui procedimentos peculiares, envolvendo a preparação dos dados para a análise, visto que esse processo "consiste em extrair sentido dos dados de texto e imagem" (CRESWELL, 2007, p. 194).

A análise dos dados abrange várias etapas, a fim de que se possa conferir significação aos dados coletados

(ALVES-MAZZOTTI;

GEWANDSZNAJDER, 1998; CRESWELL, 2007; FLICK, 2009; MINAYO, 2001). A análise dos dados primários e secundários foi realizada pelo método análise de conteúdo, com abordagem qualitativa e categorização temática de dados, como recomendam Bardin (1991) e Minayo (2008). Conforme Flick (2009), este método de análise é bastante utilizado quando o estudo envolve a coleta de dados/evidências resultantes de entrevistas em profundidade. Segundo Minayo (2008), existem diferentes tipos de análise de conteúdo, incluindo: de expressão, de avaliação, de relações, de enunciação e de categorial temático. O presente estudo aborda a última categoria e teve como objetivo descobrir os núcleos de sentido que compõem uma comunicação que tenha real significado para o objetivo a ser analisado, trabalhando de forma mais interpretativa ao invés de inferências estatísticas.

Conforme recomenda Yin (2001), no método estudo de caso alguns critérios de validação devem ser empregados para assegurar a qualidade e credibilidade dos resultados. Nestes estudos, a avaliação da validade deve ser feita à luz do propósito da investigação e pode envolver a aplicação de testes de validade de constructo, validade instrumental e/ou validade interna, dentre outros. Em última análise, esta validação define a utilidade do estudo realizado e de suas proposições e/ou resultados com relação ao problema investigado. Assim, no âmbito deste estudo, haverá preocupação em verificar a validade de constructo, instrumental e interna do estudo de caso a ser aplicado. A validade do construto diz respeito ao estabelecimento de medidas de operação adequadas para os conceitos teóricos a serem estudados. Para isso, o referencial teórico buscou abordar os constructos relevantes no entendimento do fenômeno/objeto analisado e fundamentado em autores/estudos alinhados com o problema de pesquisa.

\subsection{Instrumento de intervenção - Yield Management}

Autores como Jones e Hamilton (1992), Kimes (1989), Jauncey, Mitchell e Slamet (1995) Treszl (2012), Van Ryzin (2004), Lieberman (2005), Wirtz et al. (2003), expõe em suas pesquisas 
características que as empresas que realizam YM possuem em comum.

Harris e Peacock (1995), por exemplo, oferecem uma lista de dez requisitos divididos entre motivadores e habilitadores para a empresa que pensa em adotar o sistema. Os motivadores indicam se a empresa possui os requisitos para a adoção e os habilitadores se a empresa está capacitada para a adoção.

Desta forma, o primeiro passo, para a implantação do YM é analisar se a instituição atende as condições favoráveis apontadas pelos respectivos autores.

\section{Requisitos Motivadores:}

- Capacidade fixa: As decisões de preço, pelo menos no curto prazo, são tomadas considerando-se as restrições de capacidade;

- Capacidade perecível: A não utilização da capacidade em um determinado momento representa a sua perda, por ser impossível estocá-la para sua utilização posterior;

- Incerteza da demanda: Quando houver incerteza quanto ao comportamento da demanda, é necessária a adoção de sistemas para sua previsão. Os gerentes podem se antecipar e se preparar melhor para tomar as devidas ações. Para isso, devem sistematicamente guardar informações a respeito dos padrões históricos da demanda, além de processar e analisá-los para estabelecer tendências, ao longo do tempo;

- Custos fixos elevados: Empresas que possuem custos fixos elevados e custos incrementais pequenos para atender uma unidade a mais, levam os administradores a considerarem a possibilidade de oferecer descontos.

\section{Requisitos Habilitadores:}

- Mercados segmentáveis: Um primeiro passo essencial a ser tomado pela administração é determinar se o mercado pode ser segmentado de forma eficaz. A empresa deve distinguir os segmentos do mercado onde está atuando para que possa selecionar os segmentos de interesse e identificar oportunidades de diferenciação de preço;

- Facilidade de prevenir arbitragem: Ao segmentar o mercado, as empresas devem atentar para a possibilidade dos seus produtos de menor qualidade, destinados a segmentos sensíveis a preço, serem adquiridos a preços mais baixos, e a seguir, serem revendidos para os segmentos mais nobres como se fossem produtos de alta qualidade;

- Necessidade de reservas adiantadas: As empresas possuem uma grande vantagem na hora de realocarem sua capacidade ou reajustarem preços quando o sistema de pagamento ou reservas adiantadas é uma prática do setor;

- Bancos de dados de vendas: As empresas que planejarem e desenvolverem os seus bancos de dados de vendas terão uma vantagem competitiva, pois podem verificar $\mathrm{O}$ comportamento de seus clientes a variações no preço, dias da semana, estações do ano etc.;

- Previsão: Quem fizer uma previsão mais precisa das vendas obterá vantagem, uma vez que será mais fácil se adequar às alterações na economia, no comportamento dos consumidores ou no comportamento da concorrência;

- Desenvolver um sistema de vigilância dos concorrentes: O sistema de Yield Management requer ajustes rápidos a mudanças inesperadas do mercado. Para isso deve monitorar as atividades da concorrência, assim como os ganhos e perdas de clientes.

Uma vez satisfeitos estes requisitos, Harris e Peacock (1995) entram nos aspectos operacionais de implantação e listam os passos necessários para colocar em prática o sistema:

- Definir os segmentos de mercado: O primeiro passo é determinar se a segmentação do mercado é viável. Uma análise de segmentação leva a organização a entender o que os clientes estão comprando, como eles compram, o que eles valorizam, e quanto eles estão dispostos a pagar. Seu objetivo é descobrir correlações entre a disposição a pagar e as características do segmento e explorar esse comportamento com a intenção de aumentar as receitas;

- Dentro de cada segmento especificar as classes de serviço e as condições para participar das classes. Duas ou três classes podem ser suficientes, mas dependendo do negócio, dez ou quinze pode ser um número manejável. Um exemplo é dividir as classes em "premium"," "preço total" e "desconto";

- Estabelecer o período que o sistema irá prever. $\mathrm{Ou}$ seja, cada segmento tem suas características, possuindo uma determinada sensibilidade ao tempo. Essa etapa determina o número de dias antes da venda de um serviço ou unidade da capacidade. $\mathrm{Na}$ indústria da aviação, o gerenciamento da disponibilidade de assentos começa trezentos dias antes da data do voo. Em hotéis, normalmente começa seis meses antes da ocupação;

- Ajustar os preços para cada classe de serviço. É importante que se façam pesquisas para os ajustes de preços, sendo que esses podem ser definidos pelo conhecimento da sensibilidade 
aos preços que determinado segmento de clientes apresenta. Além disso, os preços também podem ser ajustados a partir de um conjunto de valores hipotéticos por classe e, em seguida, levantam-se amostras representativas de consumidores para determinar sua receptividade a diferentes preços. Uma outra alternativa é a de projetar um experimento formal com diferentes níveis de preços. Esta última abordagem tem o melhor potencial de produção de dados com um alto grau de validade, mas será certamente a mais cara e demorada dos três;

- Coletar informações sobre como cada classe está respondendo aos diferenciadores. As empresas interessadas em usar táticas de YM deve continuamente identificar novos diferenciais. Tal como acontece com outros sistemas de decisão com base em previsão, o YM requer uma certa quantidade de ajustes para calibrar os modelos aos dados reais;

- Especificar o tamanho relativo de cada classe de serviço. As classes de serviços apresentam diferenças significativas nas suas capacidades de reserva. Para cada diferenciador pode ser estabelecida tamanhos de classe de reservas diferentes;

- Estabelecer as curvas limite para as dimensões das classes de serviços;

- Deve-se criar um caminho de crescimento esperado para cada classe de reserva, com curva superior, limite inferior em todo o caminho de crescimento esperado. A trajetória de crescimento é uma previsão da forma como os compromissos são feitos para aquisição de unidades ao longo do tempo. As curvas de limiar mostram se os compromissos para o serviço estão no caminho certo. Se as reservas estiverem dentro das curvas estabelecidos, então não se justifica nenhum ajuste dinâmico para o tamanho do segmento;

- Desenvolver um sistema de monitoramento dos concorrentes. Para manter a sensibilidade do sistema de YM, os concorrentes devem ser monitorados. Isso significa acompanhar regularmente os preços do mercado. O sistema deve ser configurado para detectar qualquer alteração de ganhos e perdas de clientes.

\section{APRESENTAÇÃO DO CASO}

Esta seção contextualiza a Universidade Alfa e aborda sua estrutura organizacional, missão, visão e valores. Além disso, caracteriza o Curso de Administração a Distância, apresentando o problema da instituição, foco do estudo.

\subsection{A universidade Alfa}

Esta pesquisa foi realizada na Universidade Alfa, uma instituição comunitária multicampi orientada para a produção da educação, ciência, cultura e para o desenvolvimento social. É uma universidade de caráter comunitário, sem fins lucrativos.

A Universidade Alfa possui três Campi, denominados Campus Sul, Campus Norte e Campus Virtual. No Campus Sul o número de colaboradores é de 600 (seiscentos) profissionais, e 13.000 (treze mil) alunos; no Campus Norte, o número de colaboradores é de 500 (quinhentos) profissionais, e 12.000 (doze mil) alunos; e no Campus Virtual o número de colaboradores é de 400 (quatrocentos) profissionais e 25.000 (vinte e cinco mil) alunos.

A escolha desta IES justifica-se pelo fato da Alfa ser reconhecida como uma das maiores ofertantes de EaD do país. No Ranking Internacional Cybermetrics Lab 2013 - a Alfa está entre as 56 melhores universidades brasileiras no que tange à disseminação do conhecimento na web. Em 2013 contava com 25.000 (vinte e cinco mil) alunos, tornando-se uma das mais importantes instituições do país nessa modalidade em apenas alguns anos.

Com relação à amostragem, o estudo da Alfa envolverá a coleta de dados com gestores do Curso de Administração a Distância e da AlfaVirtual. Em termos quantitativos, o tamanho da amostra será definido à medida que o pesquisador constatar que a quantidade de entrevistas e evidências coletadas for suficiente para atender ao objetivo geral e aos objetivos específicos do estudo, considerando a população envolvida. Assim, o processo de amostragem deste estudo é por intencionalidade (julgamento do pesquisador) na seleção dos entrevistados e por saturação teórica (ou saturation sampling) na definição do tamanho amostral.

\section{2 $O$ curso de Administração a distância}

O projeto de educação a distância da Alfa inclui o adequado planejamento de infraestrutura física e, principalmente, tecnológica, que é desenvolvida objetivando o atendimento das necessidades pedagógicas e de comunicação entre professor, aluno e Instituição. Os cursos contam com os serviços de atendimento estruturados pela Universidade para suporte e atendimento aos estudantes, dentre eles destacam-se: Secretaria de ensino, Secretarias de apoio, SAIAC, Laboratórios, Biblioteca, Polos de apoio presencial e demais serviços de atendimento on-line disponibilizados no Portal Alfa. Por meio do Espaço Virtual de Aprendizagem (EVA), aos professores cabe a responsabilidade pela gestão pedagógica, atualização dos conteúdos e metodologias e interagindo de maneira democrática com os acadêmicos.

$\mathrm{Na}$ modalidade a Distância a secretaria de Ensino inicia o registro acadêmico de cada aluno a partir do envio da documentação exigida para ingresso. 
Toda a documentação recebida é cadastrada no Sistema Acadêmico, bem como a classificação de cada candidato. Após a classificação, é feita a matrícula dos candidatos classificados e a partir da confirmação da matrícula iniciam-se os registros de inclusão dos alunos matriculados nas turmas no Sistema Acadêmico, registro de notas, conferência dos dados pessoais, expedição da documentação solicitada pelo aluno, como declarações, históricos entre outros.

Como sala de aula, os estudantes utilizam o Espaço Virtual de Aprendizagem (EVA). O EVA oferece toda a estrutura necessária de suporte técnico e pedagógico no que diz respeito ao uso de multimídias, conteúdos digitais, dos acervos bibliográficos (livros, revistas, entre outros materiais) e dos espaços de trocas de aprendizagem com professores, tutores e com os colegas. Além da estrutura física da sede e do Espaço Virtual de Aprendizagem o curso também conta com a estrutura física dos polos para o atendimento presencial aos estudantes.

\subsection{Contextualizando o problema da instituição de ensino}

Hoje as instituições de ensino superior que ofertam cursos, tanto na modalidade presencial como a distância, percebem que a realidade apresentada pelo mercado é um excesso de oferta de vagas bastante superior à demanda atual. Esse fato acabou gerando o aumento de vagas ociosas na maioria das IES, em especial as particulares. O número crescente de IES oferecendo cursos a distância, o encolhimento do crescimento populacional na faixa etária de 18 a 24 anos, reduzindo a população com algum potencial de investir em formação superior (LIMA, 2006), a realidade econômica vivenciada no país, são alguns dos fatores que tem levado as instituições a rever suas estratégias e buscar novos caminhos para se manterem competitivas.

Diante desse cenário, o Curso de Administração a Distância da Universidade Alfa tem procurado oferecer uma variedade de formas de descontos para atrair novos estudantes. No entanto, avaliar se as estratégias utilizadas estão trazendo os resultados esperados é fundamental para o futuro da instituição.

Nesse sentido, as etapas que seguem trazem para o Curso de Administração uma proposta de implantação do YM. Assim, após contextualizar a instituição e o curso, passa-se para a etapa que envolve a entrevista com os gestores com o objetivo de analisar a viabilidade para sua implantação. A terceira e última etapa, engloba a proposta do uso da ferramenta pelo curso. Nesta etapa, estão presentes todos os processos que são utilizados hoje pela Alfa, assim como a proposta de utilização da ferramenta YM.

\subsection{Instrumento de Intervenção $\quad-$ Yield Management}

Essa etapa se constitui em suas seções. A primeira apresenta a entrevista realizada com os gestores do Curso de Administração a Distância e da AlfaVirtual e a segunda parte engloba a proposta de utilização do YM para o respectivo curso baseado nos estudos de Jones e Hamilton (1992).

O primeiro passo, para a implantação do YM é analisar se a instituição atende os Requisitos Motivadores - indicam se a empresa possui os requisitos para a adoção e os Requisitos Habilitadores se a empresa está capacitada para a adoção. Desta forma, os requisitos foram apresentados para os entrevistados para que eles analisassem esses aspectos sob o seu ponto de vista. Essa seção contém os dados coletados, com os depoimentos dos entrevistados, que estão identificados como entrevistados 1,2 e 3 .

\section{Requisitos Motivadores}

1. A instituição possui capacidade fixa?

Os três entrevistados entendem que a Alfa possui capacidade fixa, pois tem um número determinado de vagas. O Entrevistado 1 relata: "a capacidade fixa nós temos, pois sabemos mais ou menos quantos alunos temos que atender por ingresso, entrada, semestre, enfim. Sabemos que não podemos ultrapassar esse número por atendimento, senão teríamos que aumentar o número de funcionários na área de atendimento, matrícula, produção de materiais, pois temos um limite de atendimento que acabamos não chegando, mas tem uma capacidade fixa sim". O entrevistado 2 afirma que "a instituição tem capacidade fixa por ter um limite de vagas" e o entrevistado 3 pontua que "existe a capacidade fixa, no entanto não há variações de preços. No máximo temos políticas comerciais que atendem determinados grupos, mas não há essa flexibilidade para alterar mensalidades em função do que acontece no mercado".

\section{A capacidade da instituição é perecível?}

Os entrevistados expõem que a capacidade da instituição é perecível. O Entrevistado 1 acredita que sim, pois "se você não encher a sala, você está pagando os professores do mesmo jeito. Mesmo sendo EaD, é perecível". Para o Entrevistado 2: "Sim, a capacidade é perecível e não estocável. Começou o semestre as vagas não preenchidas se tornarão ociosas". O Entrevistado 3 pontua que é perecível.

\section{Existe a incerteza da demanda?}

Os três entrevistados entendem que a demanda do mercado é incerta. Para o Entrevistado 1: "Existe a demanda incerta sim, principalmente por ser mercado nacional, o mercado regional tem controle total. Hoje temos grupos internacionais de educação que investem milhões na área e adquirem aquisições de grupos, surgem novas concorrências e vem com preços 
atraentes. A qualidade não conhecemos, mas o preço é atraente, é diferente do presencial que tem que ter instalações [...] a primeira coisa que o aluno vê é realmente o preço, pois não tem uma estrutura para se encantar". Os entrevistados 2 e 3 relatam que o curso possui um acompanhamento histórico de inscrições e matrícula, baseado em série histórica e estas são acompanhadas e estudadas.

4. Os custos fixos são elevados?

Os custos fixos do EaD são muito elevados, principalmente as fases iniciais, segundo os três entrevistados. "Juntando o atendimento e pessoal são mais de 130 funcionários trabalhando só para $\mathrm{EaD}$. Além disso, tem mais os professores e coordenadores, é muita gente. A estrutura toda, os polos, os atendimentos, tudo isso envolve muitas pessoas".

\section{Requisitos Habilitadores}

5. O mercado é segmentável?

Os entrevistados entendem que o mercado do curso é segmentável, sendo que os entrevistados 1 e 3 expõem que uma das formas de segmentar os alunos seria por região.

adiantadas?

6. Existe a necessidade de reservas

Em relação as reservas antecipadas, o Entrevistado 1 diz que "a inscrição é feita com antecedência. Sendo que a etapa de inscrição passa por um processo que envolve o encaminhamento de documentação e a promulgação dos documentos para depois efetivar a matrícula. Porém, no decorrer desse processo a instituição vai perdendo alunos em todas as etapas. Ou seja, a matrícula não chega nem a $30 \%$ ou $40 \%$ dos alunos que fizeram a inscrição no início. O aluno se perde por alguns motivos como pelo fato da inscrição não ser paga ou pela falta da documentação exigida". O Entrevistado 2 acredita que "não existe a necessidade de reserva adiantada, porque sempre sobra vaga em todas as instituições. Não é como o curso de medicina que tem 50 candidatos por vaga, hoje não tem mais isso, pois é só apresentar o histórico escolar e o aluno entra. Ele só vai perder a vaga se não apresentar algum documento legal". O Entrevistado 3 relata que os alunos fazem a reserva por meio da inscrição, o problema é a morosidade do processo que pode levar até três meses e nesse período muitos alunos acabam desistindo do curso.

7. Utilizam bancos de dados de vendas?

Os três entrevistados afirmam que sim. O Entrevistado 1 afirma que "sim, mas não é específico para cada curso. A AlfaVirtual tem, e por meio dele conseguem acompanhar o comportamento do aluno online. A divulgação das inscrições do curso de administração tem algumas informações como: quantos alunos clicaram, quais os links, onde navegaram, o que mais os atraiu na página". O Entrevistado 2 acrescenta "há a utilização do Business Intelligence, não com banco de dados, mas com planilhas, pesquisas com alunos, com dados e pesquisa de evasão".

\section{Realizam previsão?}

O curso realiza previsão, segundo os três entrevistados. $\mathrm{O}$ entrevistado 1 complementa relatando que "as previsões são feitas como acompanhamento dos resultados das divulgações que caso não deem retorno, os meios de comunicação são acionados. Além disso, as previsões de mercado têm levado em conta a realidade da economia que estamos vivendo e já estão trabalhando para o ano que vem e visualizando o que vai acontecer para os cursos do EaD”. O Entrevistado 2 pontua que "desde 2007 a instituição está monitorando o mercado, a economia, a concorrência. Pois, é por meio do monitoramento que fazemos as previsões e trabalhamos a partir delas". O Entrevistado 3 relata que previsões fazem parte do cotidiano do curso. concorrente?

9. Possuem algum sistema de vigilância

A instituição faz o monitoramento constante da movimentação da concorrência. Para o Entrevistado 1: "Existe Monitoramento da concorrência não por curso, mas por instituição. É feito de forma semestral pelos boletins do EaD por instituição e pelo censo da Abed anual do EaD. Estamos sempre antenados nos novos entrantes, se tem algum curso novo e se os preços são diferenciados". De acordo com o Entrevistado 2: "Sim, constantemente". O Entrevistado 3 acrescenta que acompanham a concorrência, "mas implementar mudanças baseadas nos dados apresentados, é outra história".

Diante dos resultados da pesquisa sobre os requisitos motivadores e habilitadores, foi possível constatar que a instituição de ensino possui todos os requisitos necessários para a implantação da ferramenta YM. Desta forma, o estudo segue com uma proposta baseada na implantação do YM no Curso de Administração a Distância, tendo como objetivo apresentar aos gestores uma forma nova de trabalhar o equilíbrio entre oferta e demanda com o intuito de maximizar receita.

\subsection{Proposta de utilização do YM para o curso de Administração a Distância da universidade Alfa}

Os resultados das pesquisas realizadas sobre o YM mostram que essa ferramenta tem sido utilizada principalmente em companhias aéreas, hotéis e está se espalhando entre setores como entretenimento, aluguel de carros, restaurantes, dentre outros. No entanto, foi possível observar que não existem pesquisas ou estudos que tenham como foco a utilização do YM no setor da educação.

Nesse sentido, esta pesquisa traz uma proposta da utilização do YM adaptada para o Curso de Administração a Distância da Alfa, tendo como base os estudos de Jones e Hamilton (1992) que apontam sete 
Yield Management em Instituições de Ensino Superior: Um Estudo De Caso na Modalidade de Ensino a Distância de uma Universidade Comunitária

passos para o sucesso da implantação de um modelo de sistema de YM. A Figura 1 representa graficamente estes passos e destaca os principais tópicos desenvolvidos em cada passo.

Figura 1 - Passos para implantação e acompanhamento do YM no curso de Administração a distância da universidade Alfa

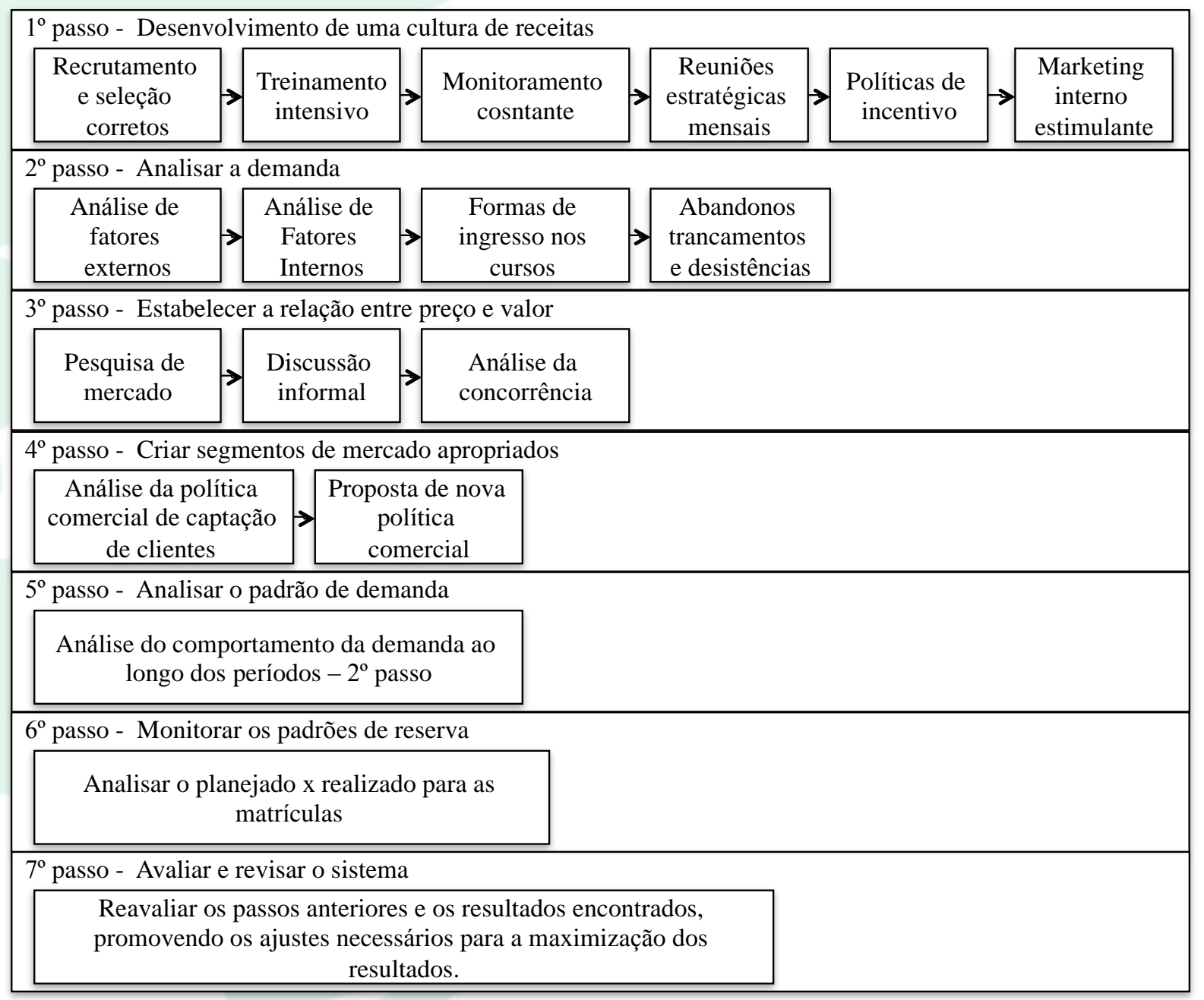

Fonte: Autores da pesquisa, 2015.

O primeiro passo foca no desenvolvimento de uma cultura de receitas na IES, para tal Centurión (2014) propõe algumas ações: o recrutamento e seleção correto dos profissionais que atuarão na implantação do marketing para o YM; o treinamento intensivo da equipe que deve estar familiarizada com a filosofia de maximização de receitas; a criação de metas específicas que proporcionem a visualização da evolução dos resultados com a implantação do YM; reuniões estratégicas que envolvam previsão de demanda, segmentação de clientes e gestão dos canais de distribuição; a promoção de políticas de incentivo; por meio de um marketing interno ativo estimular e valorizar colaboradores que destacam-se nas estratégias implantadas.
O segundo passo dá ênfase para a análise da demanda que ocorre em dois momentos: (i) a análise de fatores externos utilizando a ferramenta PESTAL que considera a análise política, econômica, social, tecnológica, ambiental e legal; avaliando os fatores de risco considerados em cada grupo, representados no Quadro 1. A segunda análise enfatiza os fatores internos por meio de entrevistas com os gestores da Alfa Virtual e do Curso de Administração a Distância. No segundo momento é feita a análise da demanda propriamente dita, em que se propõe para o caso o levantamento dos dados históricos das formas de ingresso no curso nos últimos três anos, além dos abandonos, trancamentos e desistências no mesmo período. 
Yield Management em Instituições de Ensino Superior: Um Estudo De Caso na Modalidade de Ensino a Distância de uma Universidade Comunitária

Quadro 1 - Análise Pestal da Alfa Virtual

\begin{tabular}{|c|c|}
\hline Fatores & Fatores de risco \\
\hline \multirow{6}{*}{ Fatores Políticos } & Política econômica \\
\hline & Política de taxas e impostos \\
\hline & Programas e atitudes do governo \\
\hline & Estabilidade política \\
\hline & Políticas de educação \\
\hline & Políticas de infraestrutura \\
\hline \multirow{6}{*}{ Fatores Econômicos } & Crescimento econômico \\
\hline & Taxas de juros e política monetária \\
\hline & Despesas do governo \\
\hline & Inflação \\
\hline & Taxa de câmbio \\
\hline & Taxa de desemprego \\
\hline \multirow{7}{*}{ Fatores Sociais } & Distribuição de renda \\
\hline & Taxa de crescimento da população \\
\hline & Distribuição da idade \\
\hline & Mudanças no estilo de vida \\
\hline & Atitudes do trabalhador \\
\hline & Grau de instrução \\
\hline & Consciência da saúde e segurança \\
\hline \multirow{4}{*}{$\begin{array}{l}\text { Fatores } \\
\text { Tecnológicos }\end{array}$} & $\begin{array}{l}\text { Novos produtos e serviços } \\
\text { Ciclo de vida da tecnologia }\end{array}$ \\
\hline & Mudanças na tecnologia da informação \\
\hline & Mudanças na internet \\
\hline & Mudanças na tecnologia móvel \\
\hline \multirow{3}{*}{ Fatores Ambientais } & Regulamentos e proteções ambientais \\
\hline & Aspectos demográficos \\
\hline & Clima \\
\hline \multirow{6}{*}{ Fatores Legais } & Legislações específicas \\
\hline & Código de Defesa do Consumidor (CDC) \\
\hline & Leis trabalhistas (CLT) \\
\hline & Órgãos fiscalizadores \\
\hline & Regulamentos entre concorrência \\
\hline & Regulamentos de segurança \\
\hline
\end{tabular}

Fonte: Campos (2014).

Para o terceiro passo os pontos a serem levantados tem por objetivo estabelecer uma relação entre preço e valor. A investigação passa por identificar como o cliente percebe o valor dos serviços oferecidos. $\mathrm{O}$ instrumento identificado como adequado para o levantamento dessas informações é a pesquisa de mercado que deve ser realizada identificando os níveis de qualidade do curso. Estes devem ser mensurados e comparados com a concorrência em conjunto com valores cobrados. Por fim, a coleta de informações por meio de discussões informais internas em conjunto com os demais dados levantados irão formar a noção de valor do curso oferecido.

Já no quarto passo as informações coletadas nos passos anteriores servem de insumo para estabelecer quais os potenciais segmentos de mercado para atuar, verificando sua composição, tamanho, elasticidade-preço e necessidades específicas. Neste momento são analisadas as políticas comerciais de captação de clientes da IES e, identificados os segmentos com melhor potencial, apresenta-se uma nova proposta de atuação para cada segmento.

O quinto passo remete a análise do padrão da demanda a partir da nova proposta implantada no passo anterior. Os dados para análise são os mesmos levantados nos dois últimos itens do segundo passo: a forma de ingresso no curso e os abandonos, trancamentos e desistências.

O sexto passo é crítico quanto ao aspecto agilidade, pois o monitoramento do comportamento da demanda nas matrículas leva a novas estratégias para o alcance dos resultados desejados. Neste passo o 
planejado x realizado é acompanhado praticamente em tempo real. De acordo com os resultados parciais das matrículas efetivadas, comparados com as expectativas dia a dia até a data final para a efetivação das matrículas, as ações de promoção são modificadas.

O sétimo e último passo propõe a reavaliação dos passos anteriores e os resultados obtidos, promovendo correções e melhorias nos pontos que apontam fragilidades e, por outro lado, destacando os casos de sucesso e oferecendo incentivos as pessoas envolvidas no processo. Como forma de realizar está reavaliação algumas perguntas podem ser realizadas para realinhar os objetivos da Instituição:
- Os segmentos de mercado estão respondendo de acordo com o que foi planejado?

- A demanda está de acordo com a que foi prevista?

- Os gestores envolvidos no processo estão respondendo continuamente ao desafio do YM?

- Houve uma maximização da receita?

- Houve diminuição da taxa de evasão?

Para resultados de destaque é sugerida introdução de formas de incentivo aos colaboradores envolvidos no processo. O Quadro 2 traz algumas formas de incentivo aos colaboradores da instituição.

Quadro 2 - Formas de incentivo aos colaboradores

\begin{tabular}{|c|c|}
\hline Conquistas & \multicolumn{1}{c|}{ Formas de incentivo } \\
\hline Aumento do IGC & $\begin{array}{l}\text { Comunicado de parabéns pela reitoria a todos os envolvidos. } \\
\text { - } \\
\text { Encontro comemorativo, almoço ou jantar para a equipe. } \\
\text { Bônus financeiro. }\end{array}$ \\
\hline Aumento no número de matrículas & $\bullet \quad \begin{array}{l}\text { A cada semestre quando o aumento de matrículas ultrapassar } \\
\text { o semestre anterior a equipe ganha um prêmio (passeio de } \\
\text { escuna, diária em um hotel, etc.) }\end{array}$ \\
\hline Boas ideias para captação de alunos & $\bullet \begin{array}{l}\text { Incentivar boas ideias entre os colaboradores, premiando as } \\
\text { melhores. }\end{array}$ \\
\hline
\end{tabular}

Fonte: Autores da Pesquisa.

O Yield Management não é um programa de computador, na verdade, é sim uma técnica de apoio ao gerenciamento e que deve ser visto como uma forma de pensar compartilhada por toda a empresa. Nesse sentido, alguns fatores devem ser levados em consideração ao adotá-lo: é preciso que seja realizada com seriedade, pois se não for, melhor não fazê-lo; faça gradualmente e tenha certeza de que você pode implementar sua estratégia de preços; não transforme seus sonhos em pesadelos e comunique-se sempre; nunca fique satisfeito.

\section{CONSIDERAÇÕES FINAIS}

Para melhorar sua competitividade as instituições de ensino superior passaram a utilizar mecanismos para mensurar e monitorar o desempenho dos processos e da organização como um todo. A avaliação de desempenho é a ferramenta de gestão utilizada com este propósito.

A fim de aumentar a demanda e maximizar receita as IES devem buscar ferramentas que auxiliem os gestores na tomada de decisões buscando alavancar a inovação e o diferencial competitivo tanto para sua estrutura organizacional como para os serviços que oferece. Os benefícios de uma implementação de Yield
Management como forma de trabalhar o equilíbrio entre a oferta e a demanda tem atraído muitas empresas de serviços para essa forma de gestão.

Nesse sentido, este estudo buscou responder a seguinte pergunta de pesquisa: Como o Yield Management pode ser utilizado para otimizar a receita da modalidade de ensino a distância de uma IES comunitária?

Para alcançar o objetivo geral dessa pesquisa de propor a utilização do Yield Management como ferramenta para a otimização de receitas da modalidade de ensino a distância de uma IES comunitária buscouse desenvolver os seguintes objetivos específicos: 1) Contextualizar a instituição de ensino, foco do estudo, entendendo o ambiente; 2) Analisar se a modalidade de ensino a distância da instituição atende os requisitos necessários para a adoção do Yield Management; 3) Propor os passos para a implantação do YM na instituição

Com relação à contextualização da instituição, levantaram-se informações existentes na organização, como estrutura organizacional, missão, visão, valores, assim como a contextualização do Curso de Administração tanto na modalidade presencial quanto a distância. Essas informações permitiram um maior entendimento e conhecimento sobre a instituição, sua 
trajetória e seus objetivos para dar subsídios para as propostas realizadas pelo respectivo estudo.

Quanto aos requisitos necessários para adoção do Yield Management, os entrevistados pontuam que o curso possui todas as características para a adoção tanto dos requisitos Motivadores quanto dos requisitos Habilitadores. Nesse sentido, afirmam que o curso possui capacidade fixa mesmo sendo à distância, ofertando 250 vagas a cada semestre e que seus custos fixos são elevados, pois a partir do momento que uma turma inicia, as vagas que não foram preenchidas são perdidas, ou seja, sua capacidade é perecível. O setor da educação, segundo os entrevistados, também é permeado de incertezas quando o assunto é a demanda, pois mesmo tendo um acompanhamento constante de informações a respeito da concorrência, das vendas, da economia, os gestores não tem a certeza do que acontecerá nos semestres que estão por vir. Ressalta-se que em apenas um item "necessidade de reservas antecipadas", um dos entrevistados pontuou que como sobram vagas, hoje não há a necessidade de fazer reservas. Apesar de não haver essa necessidade, muitos alunos ainda fazem sua inscrição com o intuito de reservar sua vaga. No entanto, todos os gestores relatam que o processo de inscrição é extremamente moroso, pois, além de demorar em média três meses até a sua finalização, o aluno nesse período não precisa pagar nenhuma quantia. Assim, esses fatores acabam facilitando e contribuindo de maneira bastante significativa para a perda de alunos inscritos.

Como resultado para a pesquisa, a confirmação dos Requisitos Motivadores e Habilitadores possibilitou a realização da proposta do Yield Management para o Curso de Administração a Distância.

Quanto à proposta de utilização do Yield Management (objetivo específico 3), esta foi baseada nos estudos de Jones e Hamilton (1992), abordando sete passos para sua implantação. Dentro de cada passo são apresentados dados a serem levantados na instituição, referentes aos últimos três anos de exercício, como: históricos de ingresso de alunos, formas de comercialização dos serviços, dados sobre evasão, concorrência. Essas informações são fundamentais para dar subsídios para a implantação de novas estratégias por meio da ferramenta YM.

Para a implantação do YM bem-sucedido é preciso entender que as pessoas são a parte principal de todo o processo, pois, por mais que se tenham sistemas sofisticados e inúmeros dados históricos da demanda, da concorrência, do mercado, pouco acontecerá se as pessoas envolvidas não forem valorizadas. Assim, a proposta traz a importância de se ter inicialmente ao menos um colaborador da área de marketing para gerir o YM.

Cabe ao gestor responsável uma leitura constante sobre o comportamento da oferta e da demanda. Essa leitura deve ser feita observando tanto os históricos referentes ao curso, preço, mudanças no mercado, na concorrência, nas leis, na economia.
Enfim, o gestor deve ser ágil e se antecipar a todas as mudanças que possam surgir.

Uma constatação da pesquisa por meio das entrevistas foi que apesar de haver um interesse dos gestores em novas estratégias, principalmente as que se relacionam a preço, esses se mostraram incrédulos a mudanças, pois relataram que as decisões ainda estão muito centradas em poucas pessoas que não vivenciam a realidade do curso e por isso, muitas vezes, tomam decisões pautadas nas suas próprias percepções de mercado. No YM, as decisões são pautadas por uma equipe ou um gestor qualificado que levam em consideração todas as análises que envolvem o movimento da demanda e oferta. Não é simplesmente uma questão de dar descontos, mais sim procurar preencher as vagas ociosas com os alunos que são mais sensíveis ao preço, oferecendo, por exemplo, bolsas e descontos.

O melhor caminho para a mudança de um país que deseja crescer por meio da justiça e da igualdade é dar educação de qualidade para seus cidadãos. A educação transforma vidas. O YM vem ao encontro de duas necessidades do mercado: a de trazer mais alunos para as salas de aula e maximizar receita e a de ajudar a melhorar a vida de tantas pessoas que ainda sonham em poder frequentar uma instituição de ensino superior. $\mathrm{O}$ equilíbrio é o ponto chave dessa ferramenta que vem ao encontro da realidade do mercado.

Este estudo representa uma contribuição teórica relevante para o tema. Contribuiu para ampliar o conhecimento acadêmico sobre o tema Yield Management em Instituições de Ensino Superior, tendo em vista seu ineditismo. Os resultados do estudo apontaram que o Curso de Administração a Distância da Alfa possui os requisitos para adoção da ferramenta e esse fato abre novas possibilidades para futuros estudos sobre o tema. Para estudos futuros propõe-se a implantação e execução dos passos apresentados com a formatação de uma proposta de ação de implantação do YM na IES estudada.

\section{REFERÊNCIAS}

Antons, C. M., Maltz, E. N. (2006). Expanding the role of institutional research at small private universities: A case study in enrollment management using data mining. New Directions for Institutional Research, 2006(131), 69-81.

Bana e Costa, Carlos A. (1993) Três convicções fundamentais na prática do apoio à decisão. Pesquisa Operacional, 13(1), 9-20.

Bitran, G., Caldentey, R. (2003). An overview of pricing models for revenue management. Manufacturing \& Service Operations Management, 5, 203-229. 
Bobb, L. M., Veral, E. (2008). Open issues and future directions in revenue management. Journal of Revenue \& Pricing Management, 7(3), 291-301.

Brasil. Inep - Instituto Nacional de Estudos e Pesquisas em Educacionais Anísio Teixeira. (2014). Censo da educação superior 2012: resumo técnico. Brasília, DF : INEP.

Brotherton, B., Mooney, S. (1992). Yield management: progress and prospects. International Journal of Hospitality Management, 11, 23-32.

Buss, C., Parker, J., Rivenburg, J. (2004). Cost, quality and enrollment demand at liberal arts colleges. Economics of Education Review, 23(1), 57-65.

Choi, S., Mattila, A. S. (2004). Hotel revenue management and its impact on customers' perceptions of fairness. Journal of Revenue \& Pricing Management, 2(4), 303-314.

(2005). Impact of information on customer fairness perceptions of hotel revenue management. Cornell Hotel \& Restaurant Administration Quarterly, 46(4), 444-451.

Collins, M., Parsa, H. G. (2006). Pricing strategies to maximize revenues in the lodging industry. International Journal of Hospitality Management, 25, 91-107.

Cross, R. G., Higbie, J. A., Cross, D. Q. (2009). Revenue management's renaissance: a rebirth of the art and science of profitable revenue generation. Cornell Hospitality Quarterly, 50(1), 56-81.

Cross, K. F., Lynch, R. L. (1998). The SMART way to define and sustain success. National Productivity Review, 8(1, p 23-33.

Ensslin, L., Ensslin, S. R., Pacheco, G. C. (2012). Um estudo sobre segurança em estádios de futebol baseado na análise da literatura internacional. Perspectivas em Ciências da Informação, 17(2), 71-91.

Epple, D., Romano, R., Sieg, H. (2006). Admission, tuition, and financial aid policies in the market for higher education. Econometrica, 74(4), 885-928.

Figueiredo, K, Escobar, D. (2004). Gestão de capacidade em serviços. Rio de Janeiro: UFRJ/COPPEAD.

Friedel, J. N., Thomas, G. (2013). Differential tuition: a community college budgetary strategy. Community College Journal of Research and Practice, 37(3), 147-152.
Fu, C. (2010). Equilibrium Tuition, Applications, admissions, and enrollment in the college market. Journal of Political Economy, 122(2), 225-281.

Gallego, G., Phillips, R. (2004). Revenue management of flexible products. Manufacturing \& Service Operations Management, 6(4), 321-337.

Garrow, L., Ferguson, M. (2009). Staying ahead of the curve: using revenue management to help survive an economic downturn. Journal of Revenue \& Pricing Management, 8(2/3), 279-286.

Garrow, L., Ferguson, M., Keskinocak, P., Swannet, J. (2006). Expert opinions: current pricing and revenue management practice across U.S. industries. Journal of Revenue \& Pricing Management, 5(3), 237-247.

Garvin, D. A. (1991). How the Baldrige Award really works. Harvard Business Review.

Goulding, P.J., Leask, A. (1997). Scottish visitor attractions: revenue versus capacity. In: Yeoman, I., Ingold, A. (eds.), Yield management: strategies for the service industries, Cassell, London, 160-82.

Gu, Z, Caneen, J. (1998). Quadratic models for yield management in hotel rooms operation. Progress in Hospitality and Tourism Research, 4, 245-253.

Hartley, J., Rand, P. (1997). Conference sector capacity management. In: Yeoman, I., Ingold, A. (eds.), Yield management: strategies for the service industries, Cassell, London, 218-41.

Hillman, N. W. (2012). Tuition discounting for revenue management. Research in Higher Education, 53(3), 263-281.

Hoseason, J., Johns, N. (1998). The numbers game: the role of yield management in the tour operations industry. Progress in Tourism and Hospitality Research, 4), 97-106.

Hronec, S. M. (1994). Sinais vitais: usando medidas do desempenho da qualidade, tempo e custo para traçar a rota para o futuro de sua empresa. São Paulo: Makron Books.

Huefner, R. J., Largay, J. A. III. (2008). The role of accounting information in revenue management. Business Horizons, 51(3), 245-255.

Jallat, F., Ancarani, F. (2008). Yield management, dynamic pricing and CRM in telecommunications. Journal of Services Marketing, 22(6), 465-478. 
Kaplan, R. S., Norton, D. P. (1992). The balanced scorecard: measures that drive performance. Harvard Business Review, 69(1), 71-79.

(1996). The balanced scorecard as a strategic management system. Harvard Business Review, 74(1), 75-85.

(2004). Measuring the strategic readiness of intangible assets. Harvard Business Review, 82(2), 52-63.

Keeney, R. L. (1992). Value focused-thinking: a path to creative decision-making. Cambridge: Harvard Univ.

Kimes, S. E. (1989). The basics of yield management. Cornell Hotel and Restaurant Administration Quarterly, 30, 14-19.

(2000). Revenue management on the links: applying yield management to the golf-course industry. Cornell Hotel and Restaurant Administration Quarterly, 41, 127.

Lowe, B., Alpert, F. (2007). Measuring reference price perceptions for new product categories: which measure is best? Journal of Product \& Brand Management, 16(2), 132-141.

Mathies, C., Gudergan, S. (2007). Revenue management and customer centric marketing How do they influence travellers' choices? Journal of Revenue \& Pricing Management, 6(4), 331-346.

Midwinter, A. (1994). Developing performance indicators for local government: the Scottish experience. Public Money \& Management, v 14(2), 37-43.

Milla, S., Shoemaker, S. (2008). Three decades of revenue management: What's next? Journal of Revenue \& Pricing Management, 7(1), 110-114.

Montgomery, M. (2002). A nested logit model of the choice of a graduate business school. Economics of Education Review, 21(5), 471-480.

Nassif, J. M. V., Hanashiro, M. M. D. (2003). A competitividade das universidades particulares à luz de uma visão baseada em recursos. Revista de Administração Mackenzie, São Paulo, 3(1), 95-114.

Neely, A. D., Adams, C., Kennerley, M. (2002). The performance prism: the scorecard for measuring and managing business success. London: Prentice Hall.
Ng, I. C. L. (2006). Differentiation, self-selection and revenue management. Journal of Revenue \& Pricing Management, 5(1), 2-9.

Noone, B. M., Mcguire, K. A., Rohlfs, K. V. (2011). Social media meets hotel revenue management: Opportunities, issues and unanswered questions. Journal of Revenue \& Pricing Management, 10(4), 293-305.

Nurnberg, P., Schapiro, M., Zimmerman, D. (2012). Students choosing colleges: Understanding the matriculation decision at a highly selective private institution. Economics of Education Review, 31(1), $1-8$.

Orkin, E. B. (1988). Boosting your bottom line with yield management. Cornell Hotel and Restaurant Administration Quarterly, 28, 52-6.

Richardson, R. J. (1999). Pesquisa social: métodos e técnicas. São Paulo: Atlas.

Roy, B. (1985). Méthodologie Multicritére d'Aide à la décision. Paris: Economica.

(1993). Decision science or decision-aid science? European Journal of Operational Research, 66(2), 184-203.

Sauder, M., Lancaster, R. (2006). Do rankings matter? The effects of US News \& World Report rankings on the admissions process of law schools. Law \& Society Review, 40(1), 105-134.

Schwartz, Z. (2006). Advanced booking and revenue management: Room rates and the consumers' strategic zones. International Journal of Hospitality Management, 25, 447-462.

Sztipanovits, J., Karsai, G. (1997). Model-integrated computing. Computer, 30(4), 110-111.

Tokman, M., Davis, L. A., Lemon, K. N. (2007). The WOW factor: creating value through win-back offers to reacquire lost customers. Journal of Retailing, 83(1), 47-64.

Wang, X. L., Bowie, D. (2009). Revenue management: the impact on business-to-business relationships. Journal of Services Marketing, 23(1), 31-41.

Weatherford, L. R., Kimes, S. E. (2001). Forecasting for hotel yield management: testing aggregation against disaggregation. Cornell Hotel and Restaurant Administration Quarterly, 42), 63-4.

Wirtz, J., Kimes, S. E. (2007). The moderating role of familiarity in fairness perceptions of revenue 
Yield Management em Instituições de Ensino Superior: Um Estudo De Caso na Modalidade de Ensino a

Distância de uma Universidade Comunitária

management pricing. Journal of Service Research, 9(3), 229-240.

Wirtz, J., Kimes, S. E., Theng, J. H. P., Patterson, P. (2003). Revenue management: resolving potential customer conflicts. Journal of Revenue \& Pricing Management, 2(3), 216-226.

Xia, L., Monroe, K. B., Cox, J. L. (2004). The price is unfair! A conceptual framework of price fairness perceptions. Journal of marketing, 68, 1-15. 